\title{
Role of Intact Parathyroid Hormone (iPTH) Level as an Early Predictor of Postoperative Hypocalcemia after Total Thyroidectomy for Simple Multi-Nodular Goiter
}

\author{
Mohamed KF Hamed, Amr H Afifi \\ Department of General Surgery, Ain Shams University, Egypt
}

Background: Postoperative hypocalcaemia, secondary to hypoparathyroidism, is the most common complication observed in patients who undergo bilateral thyroid resection. Although hypocalcaemia is self-limiting in most patients and does not require treatment, symptomatic hypocalcemia is of concern. The aim of this research was to identify PTH-24h post-operatively as a simple predictor of early postoperative hypocalcemia following total thyroidectomy for simple multi-nodular goiter.

Methods: Fifty patients undergoing total thyroidectomy for simple multi-nodular goiter were included in a prospective controlled study with follow up of 48 hours postoperatively for the development of hypocalcemia both clinically and biochemically correlating it to the level of serum iPTH thus allocating patients in two groups; those who developed hypocalcemia (whether clinically or biochemically) and those who didn `t.

Results: A highly significant difference between PTH percent of decline was noted for the patients who developed biochemical hypocalcaemia and those who did not.

Conclusion: Serum PTH 24-h after total thyroidectomy is a reliable early predictor of hypocalcemia.

Key words: Serum intact parathyroid hormone; hypocalcemia; total thyroidectomy.

\section{Introduction}

Thyroid surgery has a history of significant changes in the technique and the incidence of complications. Continuous developments in surgical techniques and better understanding of thyroid anatomy and pathology have increased the safety of thyroid surgery and reduced the incidence of complications. Nowadays, the rate of postoperative mortality is extremely low. The most common and potentially life-threatening complications in thyroid gland surgery are vocal cord palsy and hypocalcemia. ${ }^{1}$

Preservation of the parathyroid glands during total thyroidectomy is a major concern for an endocrine surgeon because there is no guarantee of normal postoperative parathyroid function, even if the procedure is performed for benign disease. It is especially difficult to keep the parathyroid glands intact if a tumor is large, infiltrative or if there are extensive lymph node metastasis. According to one systematic review, the median incidences of transient and permanent hypocalcemia were $27 \%$ and $1 \%$, respectively, but at the worst, these rates were as high as $38 \%$ and $3 \%$, respectively. ${ }^{2}$

Early postoperative calcium monitoring, although important, is a poor predictor of subsequent symptomatic hypocalcemia. Despite the fact that the slope of postoperative serum calcium levels correlates with the development of symptomatic hypocalcemia, its utility is limited as the results are not available until $24-48$ hours post-thyroidectomy. Other approaches to monitor and predict postoperative parathyroid function and subsequent hypocalcemia have been described in the literature. These include PTH assayed either intraoperatively or in the early postoperative period. These approaches are based on the fact that intact PTH has a short half-life of 1-4 minutes, thus allowing detection of its fall early in the perioperative period. ${ }^{3}$

The nadir for hypocalcemia typically occurs at around 24-48 hours postoperatively but may be as delayed as post-op day 4 . Therefore, detecting patients requiring calcium replacement therapy with serial calcium measurements can take multiple blood tests over several days. Placing all patients on calcium therapy unnecessarily exposes many patients to unnecessary treatment and puts them at risk for hypercalcemia. A clinical laboratory method for early prediction of postoperative hypocalcemia could, therefore, facilitate earlier implementation of treatment, and early discharge ( $\leq 24$ hours). ${ }^{4}$

In the context of escalating health care costs, a number of initiatives have focused on various ways to facilitate timely hospital discharge without compromising patient safety. The importance of a reliable measure to predict a person's relative risk for developing clinically significant hypocalcemia following thyroidectomy should not be underestimated. Besides facilitating early 
discharge in low-risk patients, identification of highrisk patients would also allow prompt prophylactic treatment. Unfortunately, the classification of patients into relative risk levels for subsequent hypocalcemia is not always straightforward. ${ }^{5}$

Because of its relatively shorter half-life, changes in parathyroid hormone (PTH) precede changes in calcium by hours. ${ }^{5}$ Intraoperative PTH has been less readily adopted for use during thyroidectomy. Among those who have used PTH as a guide for guiding management after thyroidectomy, different reports exist regarding the sensitivity and specificity of PTH for accurately predicting hypocalcemia. 6 Some studies even report seemingly contradictory results. There is no consensus about the best time to obtain PTH levels for accurately predicting a patient's risk for clinically significant hypocalcemia. It is also unclear whether the absolute value of PTH versus the percentage change from preoperative to intraoperative/postoperative levels is a better predictor for postoperative hypocalcemia. ${ }^{5}$ This study was designed to identify PTH level after 24 hours as a simple predictor of hypocalcemia following total thyroidectomy in the early postoperative period.

\section{Methods}

After taking the approval of "The ethical committee for scientific research" regarding the sample size and the ethical points, fifty (50) patients presenting at our hospital (Ain Shams University Hospitals) who were decided to have total thyroidectomy for simple multi-nodular goiter between August 2016 and August 2017, were included in this prospective study provided they had normal levels of serum calcium, phosphorus, parathyroid hormone and albumin pre-operatively and they were followedup for at least 48 hours post-operatively. Patients with co-morbidities or on chronic medications that affect serum calcium or albumin were excluded. An informed consent was taken from the patients or their first-degree relatives before being included in this study.

Neck ultrasonography was done pre-operatively to all patients and multi nodular goiter was defined as multiple nodules occupying both lobes with well defined borders, iso or hyperechoic with peripheral hypoechoic halo and vascularity. Cases with parathyroid abnormalities were excluded.

Baseline serum level of thyroid hormone, parathyroid hormone, calcium "total \&ionized" and albumin were determined for all patients.

All patients were operated upon by the same surgical team and all effort was done to identify and preserve the four parathyroid glands.

It is to be mentioned that identification of three parathyroid glands intraoperatively was satisfactory for the operative team to include the case in this study; otherwise, the case was excluded and replaced with another case fulfilling the previously mentioned criteria.

The operative team adopted the same operative technique in all the patients; starting with ligation of the middle thyroid vein (if present) followed by ligation of the upper thyroid pole in mass with the superior thyroid vessels, then, the recurrent laryngeal nerve was identified. This was followed by identification of the inferior then the superior parathyroid glands and finally, the thyroid gland was freed from its attachment before starting on the other side in the same order of steps.

During the post-operative period, all patients were monitored for symptoms suggestive of hypocalcemia e.g. circumoral or acral numbness, paresthesia, muscular spasm up to tetany and convulsions.

Serum calcium, phosphorus, albumin, parathyroid hormone assay was measured 24 hours postoperatively for all recruited patients and the \% iPTH decline $=[\mathrm{iPTH}$ preop $(\mathrm{pg} / \mathrm{ml})-\mathrm{iPTH}$ postop $(\mathrm{pg} /$ $\mathrm{ml})] /$ iPTH preop $(\mathrm{pg} / \mathrm{ml}) \times 100$.

According to the results of post-operative serum calcium, patients were allocated in one of two groups: group " $A$ " including patients who were normocalcemic post-operatively and group " $\mathrm{B}$ " including those who developed hypocalcemia.

The results were reported in line with Consolidated Standards of Reporting Trials (CONSORT) Guidelines. It is to be mentioned that interim analysis of the outcome obtained in the first five (5) then ten (10) patients was done by the ethical committee taking the approval to continue our study for the whole sample size.

\section{Results}

In this study, the post-operative level of iPTH was assessed in fifty patients undergoing total thyroidectomy for having simple multi-nodular goiter then it was correlated to the post-operative level of serum calcium trying to identify its relationship to the incidence of developing post-operative hypocalcemia.

All the data obtained was analyzed using the SPSS program.

The study included 46 females (92\%) and 4 males $(8 \%)$. The mean age of the study population was 41 years (Table 1). 
Table 1: Demographic characters of study population

\begin{tabular}{lll}
\hline Total & & No. $=\mathbf{5 0}$ \\
\hline \multirow{2}{*}{ Sex } & Female & $46(92.0 \%)$ \\
& Male & $4(8.0 \%)$ \\
\multirow{2}{*}{ Age } & Mean \pm SD & $40.92 \pm 11.71$ \\
& Range & $22-65.0$ \\
\hline
\end{tabular}

All cases included in the study didn 't experience pressure manifestations. All cases didn't have tracheal shift nor did they have a retrosternal extension for the enlarged thyroid gland.

The pre-operative biological assays are shown in Table 2.
Table 2: Comparison between PreOperative biochemical assays "Ca=Calcium, PO=phosphorus, Alb.= Albumin, Corrected Ca, PTH= parathyroid hormone".

\begin{tabular}{|c|c|c|}
\hline No. $=\mathbf{5 0}$ & & Pre-Operative \\
\hline \multirow{2}{*}{$\mathrm{Ca}$} & Mean \pm SD & $9.36 \pm 0.45$ \\
\hline & Range & $8.5-10.4$ \\
\hline \multirow{2}{*}{ PO } & Mean \pm SD & $3.44 \pm 0.38$ \\
\hline & Range & $2.7-4.4$ \\
\hline \multirow{2}{*}{ Alb. } & Mean \pm SD & $4.05 \pm 0.22$ \\
\hline & Range & $3.5-4.4$ \\
\hline \multirow{2}{*}{ Corrected Ca } & Mean \pm SD & $9.32 \pm 0.42$ \\
\hline & Range & $8.74-10.16$ \\
\hline \multirow{2}{*}{ PTH } & Mean \pm SD & $40.00 \pm 12.25$ \\
\hline & Range & $20.3-63.8$ \\
\hline
\end{tabular}

The same laboratory tests were repeated to all patients 24 hours post-operatively and then the data were analyzed and correlated Tables 3-6.

Table 3: Description of study population

\begin{tabular}{llll}
\hline \multirow{2}{*}{ No. $=\mathbf{4 0}$} & & $\begin{array}{l}\text { Normal post-op. Ca } \\
\text { (Group A) }\end{array}$ & $\begin{array}{l}\text { Hypo post-op. Ca } \\
\text { (Group B) }\end{array}$ \\
\cline { 3 - 4 } & & No. $=\mathbf{1 0}$ & $10(100.0 \%)$ \\
Sex & Female & $36(90.0 \%)$ & $0(0.0 \%)$ \\
& Male & $4(10.0 \%)$ & $39.80 \pm 6.65$ \\
\multirow{2}{*}{ Age } & Mean \pm SD & $41.20 \pm 12.72$ & $29-47$ \\
\hline
\end{tabular}

Table 4: Post-Operative Corrected Ca level in correlation to post-operative individual variants

\begin{tabular}{|c|c|c|c|c|c|c|}
\hline \multicolumn{2}{|c|}{ Post-Operative } & \multirow{2}{*}{$\begin{array}{c}\text { Group A } \\
\text { No. }=\mathbf{4 0} \\
8.74 \pm 0.37\end{array}$} & \multirow{2}{*}{$\begin{array}{c}\text { Group B } \\
\text { No. = } \mathbf{1 0} \\
7.92 \pm 0.08\end{array}$} & \multirow{3}{*}{$\begin{array}{c}\text { Test value• } \\
6.907\end{array}$} & \multirow{3}{*}{$\begin{array}{c}\text { P-value } \\
0.000\end{array}$} & \multirow{3}{*}{$\begin{array}{l}\text { Sig. } \\
\text { HS }\end{array}$} \\
\hline & Mean \pm SD & & & & & \\
\hline Ca & Range & $8.3-9.5$ & $7.8-8$ & & & \\
\hline \multirow{2}{*}{ PO } & Mean \pm SD & $3.51 \pm 0.42$ & $3.64 \pm 0.78$ & \multirow{2}{*}{-0.749} & \multirow{2}{*}{0.458} & \multirow{2}{*}{ NS } \\
\hline & Range & $3-4.5$ & $2.6-4.4$ & & & \\
\hline \multirow{2}{*}{ Alb. } & Mean \pm SD & $3.65 \pm 0.28$ & $4.02 \pm 0.20$ & \multirow{2}{*}{-3.988} & \multirow{2}{*}{0.000} & \multirow{2}{*}{ HS } \\
\hline & Range & $2.9-4$ & $3.9-4.4$ & & & \\
\hline \multirow{2}{*}{ Corrected Ca } & Mean \pm SD & $9.02 \pm 0.40$ & $7.90 \pm 0.13$ & \multirow{2}{*}{8.717} & \multirow{2}{*}{0.000} & \multirow{2}{*}{ HS } \\
\hline & Range & $8.38-9.62$ & $7.68-8$ & & & \\
\hline \multirow[t]{2}{*}{ PTH } & Mean \pm SD & $28.23 \pm 15.56$ & $7.86 \pm 5.32$ & \multirow[b]{2}{*}{4.053} & \multirow[b]{2}{*}{0.000} & \multirow[b]{2}{*}{ HS } \\
\hline & Range & $9.4-63.2$ & $2.5-15.2$ & & & \\
\hline
\end{tabular}


Table 5: Comparison between hypocalcemic and normocalcemic group regarding PTH level. NS: Nonsignificant; S: Significant; HS: Highly significant

\begin{tabular}{|c|c|c|c|c|c|c|}
\hline & \multirow[b]{3}{*}{ Range } & Group A & Group B & \multirow{3}{*}{$\begin{array}{c}\text { Test } \\
\text { value• }\end{array}$} & \multirow{3}{*}{ P-value } & \multirow{3}{*}{ Sig. } \\
\hline & & No. $=40$ & No. $=10$ & & & \\
\hline & & $8.38-9.62$ & $7.68-8$ & & & \\
\hline \multirow{2}{*}{ Pre-operative PTH } & Mean \pm SD & $40.60 \pm 12.38$ & $37.60 \pm 12.07$ & \multirow{2}{*}{0.688} & \multirow{2}{*}{0.495} & \multirow{2}{*}{ NS } \\
\hline & Range & $20.3-63.8$ & $21.4-53.2$ & & & \\
\hline Post-operative PTH & Mean \pm SD & $28.23 \pm 15.56$ & $7.86 \pm 5.32$ & \multirow{2}{*}{4.053} & \multirow{2}{*}{0.000} & \multirow{2}{*}{ HS } \\
\hline Range & $9.4-63.2$ & $2.5-15.2$ & & & & \\
\hline \multirow{3}{*}{$\begin{array}{l}\text { Percent of PTH decline } \\
\%\end{array}$} & Median & $-23.90 \%$ & $-77.82 \%$ & \multirow{3}{*}{$-4.174 \ddagger$} & \multirow{3}{*}{0.000} & HS \\
\hline & (IQR) & {$[(-53.96 \%)-(-14.07 \%)]$} & {$[(-91.04 \%)-(-65.89 \%)]$} & & & \\
\hline & Range & $(-75.39 \%)-(-0.94 \%)$ & $(-94.29 \%)-(-63.55 \%)$ & & & \\
\hline
\end{tabular}

•: Independent t-test; $\neq:$ Mann Whitney test.

Table 6: Post-operative

\begin{tabular}{lcc}
\hline \multirow{2}{*}{ Post-Operative } & \multicolumn{2}{c}{ Percent of decline } \\
\cline { 2 - 3 } & $\mathbf{r}$ & P-value \\
\hline $\mathrm{Ca}$ & $0.809^{* *}$ & 0.000 \\
Corrected Ca & $0.705^{* *}$ & 0.000 \\
PTH & $0.874^{* *}$ & 0.000 \\
\hline
\end{tabular}

\section{Discussion}

The pathogenesis of postoperative hypocalcemia is multifactorial; bad surgical technique and several other factors (e.g., preoperative low calcium levels, failure to identify parathyroid during surgery, postoperative bleeding, and wound infection) have been associated with postoperative hypocalcemia. Post-operative hypoparathyroidism is usually a result of inadvertent devascularization, excision or trauma to the parathyroid glands during surgery. The intimate relation between the thyroid and parathyroid glands and their blood supplies makes sound surgical technique essential to minimize parathyroid trauma. Other factors, however, contribute to postoperative hypocalcemia, such as hemodilution, increased urinary calcium excretion, calcitonin release, thyrotoxicosis and osteodystrophy.

Although postoperative hypocalcemia is the most common complication of total thyroidectomy, its consequences and the importance of prevention and treatment remain widely undervalued. ${ }^{7}$ Several studies pointed that impaired parathyroid function is the major cause for postoperative hypocalcemia. ${ }^{6}$ Several studies supported the use of postoperative IPTH and \% iPTH decline in predicting postoperative hypocalcemia, but the results are still inconclusive, and no definite laboratory cut point or guideline have yet been established. ${ }^{8}$

Attempts to predict the occurrence of postthyroidectomy hypocalcemia went through different stages of development. One of the earlier approaches by Husein et al. ${ }^{9}$ observing the slope of serial calcium levels during the post-operative period. They concluded that if the slope became positive (with two measurements at 6 and $12 \mathrm{~h}$ ), then the patient would have a high chance to remain normocalcemic. However, since most of studies approved that the nadir in serum calcium may not be reached until $48 \mathrm{hr}$ after surgery up to 4 days, and the serum calcium levels do fluctuate after calcium replacement, ${ }^{10}$ this approach didn ' $t$ appear to be optimal. PTH measurement-based protocols flourished, after the ultra-short half-life of PTH (1---3 min) was discovered. ${ }^{10}$ Huge discrepancies in the literature have raised questions about how many measurements of PTH are needed, what the best time for measurement is, what the optimal PTH cutoff level is. There were specific concerns about the safety of the adopted protocol and its effectiveness apart from the variation in the definitions used for hypocalcemia and hypoparathyroidism. ${ }^{11}$

Pattou et al. ${ }^{12}$ found that a postoperative PTH level of $12 \mathrm{pg} / \mathrm{mL}$ or less was a good predictor of hypocalcemia, but they did not state how long after surgery PTH values were obtained. Lombardi et al. ${ }^{13}$ found greater precision with measurements taken at 4 and 6 hours, with an overall accuracy of $98 \%$. Lam and Kerr ${ }^{14}$ reported that all patients with a PTH level less than $8 \mathrm{pg} / \mathrm{mL}$ measured 1 hour after the surgery became hypocalcemic, and all patients with a PTH level greater than $9 \mathrm{pg} / \mathrm{mL}$ did not. Higgins et al. ${ }^{15}$ demonstrated that $64 \%$ of those patients who subsequently required calcium supplementation 
had a decrease in PTH levels greater than $75 \%$ from baseline 20 minutes after surgery, and 74\% of those who did not need calcium supplementation demonstrated a decrease of less than $75 \%$ from baseline. The Australian Endocrine Guidelines, 16 published in 2007, adopted the recommendations of Lombardi and colleagues to standardize obtaining a PTH level 4 hours after a thyroidectomy. The wide variability of the predictors for the development of hypocalcemia across centers suggested that the measurement of PTH at any time in the postoperative period may be a reliable predictor of hypocalcemia. Our study suggests that obtaining a PTH level 24 hours post-operatively is adequate to predict the need for calcium and/or calcitriol supplementation after discharge from the hospital.

Most of the studies did not take into account the preoperative PTH level despite the fact that the major cause in variation of cutoff PTH values can be the variation in baseline (preoperative) PTH. The variation in baseline PTH might be secondary to population norms, or more likely to the differences in vitamin $D$ level and incidence of deficiency. ${ }^{17}$ Therefore, the use of percent of change, rather than absolute PTH values, would probably account for this variable. Al-Dhahri et al 10 tested the accuracy of post thyroidectomy calcium level and PTH level at different times post-operatively, they found that PTH measurements at $6,12,20,32$, and 44 hr had high predictive probability to detect clinical hypocalcemia; Despite the fact that Ca accuracy markedly improved in the second day, it never reached accuracy of PTH measurement. Because all PTH measurements provided high accuracy, it was clinically meaningful to be able to base the clinical decision at the earliest measurement. Therefore, they suggested using a single early PTH measurement for hypocalcemia prediction and then use $\mathrm{Ca}$ for doses adjustment. Le et al. ${ }^{18}$ evaluated the validity and safety of single 1-h PTH for predicting hypocalcemia, and they concluded that it was very reliable and safe. However, Two patients in the low risk group ( $n=94)$ became hypocalcemic, resulting in a negative predictive value of $98 \%$. Another criticism for the use of PTH measurement is its cost. In many hospitals, this test may be unavailable or take hours to have results.

A single measurement of iPTH showed conflicts with a lack of sensitivity or specificity in some studies, ${ }^{8}$ so the \% iPTH decline between its preoperative and postoperative levels was studied to solve this problem by Suwannasarn et al. ${ }^{8}$ The threshold of $\%$ iPTH decline is different in each publication, since the accuracy of a single postoperative iPTH measurement depends on the threshold considered; however, iPTH decline seems to directly reflect the individualized parathyroid function impairment in the postoperative period. ${ }^{8}$
Our study suggests that a single measurement of postoperative iPTH24hr and \% iPTH decline can reliably predict patients at risk for significant postoperative hypocalcemia. This goes in accordance with the study done by Lecerf et al., ${ }^{19}$ stating that a decline of iPTH level $>68.5 \%$ was precise for early diagnosis of hypocalcemia after total thyroidectomy. It is worth to state that we did not include the data of patients who underwent parathyroid autotransplantation.

A literature review confirmed that there is no consistent use of, rationale for, or seeming need to declare definitions of "transient" and "permanent" hypoparathyroidism. The definition of permanent hypoparathyroidism varies in terms of calcium level, need for supplementation (calcium and/or active vitamin D), and duration of hypoparathyroidism. Some studies defined permanent hypoparathyroidism as hypocalcemia with ongoing requirement for supplements, i.e., calcium and vitamin $\mathrm{D}$, or calcium and/or vitamin D. The duration of ongoing requirement for supplements also varied from $>6$ months to 12 months. Suwannasarn et al. 8 found that $19(29 \%)$ of the 65 patients had permanent hypoparathyroidism at 6 months after surgery, following the definition requiring ongoing supplementation of calcium and active vitamin D beyond 6 months. A iPTH $4 \mathrm{hr}<12.5 \mathrm{pg} / \mathrm{mL}$ and \% iPTH decline $>72 \%$ could define permanent hypoparathyroidism with equal accuracy according to their study. Moreover, they reported measurements of iPTH $4 \mathrm{hr}$ and \% iPTH decline are reliable for predicting immediate postoperative significant hypocalcemia and permanent hypoparathyroidism. They also suggested supplementation with calcium carbonate and active vitamin $D$ to be initiated early in patients with iPTH4hr $<12.5 \mathrm{pg} / \mathrm{mL}$ to prevent significant hypocalcemia, which could be symptomatic and fatal, and that an iPTH measurement protocol be implemented for post-thyroidectomy care to facilitate early discharge and supplementation in this high-risk group of patients.

Most studies revealed that lower postoperative PTH levels $30 \mathrm{~min}$ to five days after surgery was associated with transient hypocalcaemia. This association was independent of postoperative calcium levels. ${ }^{20}$ In other studies, low postoperative PTH $(<6.35 \mathrm{pg} / \mathrm{ml})$ between one hour and one day after surgery had a sensitivity ranging from $69-100 \%$ in predicting transient hypocalcaemia. A postoperative decline in PTH of more than $37-88 \%$ compared with preoperative levels has a sensitivity ranging from $70-100 \%$ for predicting transient hypocalcaemia. In patients with transient hypocalcaemia after surgery, a PTH concentration $<12 \mathrm{pg} / \mathrm{ml}$ at one month after the operation was associated with the development of permanent hypocalcemia. ${ }^{21}$ 
Lastly, it is essential to state that early postoperative PTH can accurately predict patients not at risk of hypocalcaemia. The ability to accurately predict patients not at risk of hypocalcaemia allows us to safely adopt an early discharge protocol for many of our patients. By using the PTH results to tailor oral calcium and vitamin D supplementation, we are able to better manage post-thyroidectomy hypocalcemia.

\section{Conclusion}

These results indicate that a low serum intact PTH level after thyroidectomy is an earlier predictor of hypocalcaemia than serum calcium levels alone. Serum PTH 24-h after total thyroidectomy is a reliable predictor of hypocalcaemia and can allow safe early discharge of patients from hospital. Patients with a normal postoperative serum PTH and normal serum calcium the following morning will not develop hypocalcaemia and can be considered for discharge that day. Also, PTH level was very useful in determining which patient needs oral calcium and vitamin $\mathrm{D}$ in addition.

\section{References}

1. Khafagy AH, Abdelnaby I: Total thyroidectomy: Ligasure versus Clamp \& Knot technique for intraoperative hemostasis. Egyptian Journal of Ear, Nose, Throat and Allied Sciences. 2013; 14(2): 59-65.

2. Park I, Rhu J, Woo JW, et al: Preserving parathyroid gland vasculature to reduce postthyroidectomy hypocalcemia. World Journal of Surgery. 2016; 40: 1382-1389.

3. AIQahtani A, Parsyan A, Payne R, et al: Parathyroid hormone levels 1 hour after thyroidectomy: An early predictor of postoperative hypocalcemia. Canadian Journal of Surgery. 2014; 57(4): 237-240.

4. Le TN, Kerr PD, Sutherland DE, et al: Validation of 1-hour post-thyroidectomy parathyroid hormone level in predicting hypocalcemia. Journal of Otolaryngology - Head \& Neck Surgery. 2014; 43: 5.

5. David R, Andrew M, Eric R, et al: Comparison of Intraoperative versus Postoperative Parathyroid Hormone Levels to Predict Hypocalcemia Earlier after Total Thyroidectomy. Otolaryngology-Head and Neck Surgery. 2015; 153(3): 343-349.

6. Graff AT, Miller FR, Roehm CE, Prihoda TJ: Predicting hypocalcemia after total thyroidectomy: parathyroid hormone level vs. serial calcium levels. Ear Nose Throat J. 2010; 89(9): 462-465.
7. Ozemir IA, Buldanli MZ, Yener O, Leblebici M, Eren T, Baysal H, Alimoglu O: Factors affecting postoperative hypocalcemia after thyroid surgery: Importance of incidental parathyroidectomy. North Clin Istanb. 2016; 3(1): 9-14.

8. Suwannasarn M, Jongjaroenprasert W, Chayangsu P, Suvikapakornkul R, Sriphrapradang $\mathrm{P}$ : Single measurement of intact parathyroid hormone after thyroidectomy can predict transient and permanent hypoparathyroidism: a prospective study. Asian J Surg. 2016; 00150-5.

9. Husein M, Hier MP, Al-Abdulhadi K, Black M: Predicting calcium status post thyroidectomy with early calcium levels. Otolaryngology-Head and Neck Surgery. 2002; 127(4): 289-293.

10. Al-Dhahri FS, Al-Ghonaim YA, Terkawi SA: Accuracy of post-thyroidectomy parathyroid hormone and corrected calcium levels as early predictors of clinical hypocalcemia. J Otolaryngol Head Neck Surg. 2010; 39: 342-348.

11. Mazotas IG and Wang TS: The role and timing of parathyroid hormone determination after total thyroidectomy. Gland Surg. 2017; 6(1): S38-S48.

12. Pattou F, Combemale F, Fabre S, et al: Hypocalcemia following thyroid surgery: incidence and prediction of outcome. World $\boldsymbol{J}$ Surg. 1998; 22: 718-724.

13. Lombardi $C P$, Raffaelli $M$, Princi $P$, Dobrinja $C$, Carrozza C, Di Stasio E, D'Amore A, Zuppi C, Bellantone R: Parathyroid hormone levels 4 hours after surgery do not accurately predict post-thyroidectomy hypocalcemia. Surgery. 2006; 140(6): 1016-1023.

14. Lam A, Kerr P: Parathyroid hormone: an early predictor of post-thyroidectomy hypocalcemia. Laryngoscope. 20003; 113: 2196-2200.

15. Higgins KM, Mandell DL, Govindaraj S: The role of intraoperative rapid parathyroid hormone monitoring for predicting thyroidectomy-related hypocalcemia. Arch Otolaryngol Head Neck Surg. 2004; 130: 63-67.

16. AES Guidelines 06/01 Group: Australian Endocrine Surgeons Guidelines AES06/01. Postoperative parathyroid hormone measurement and early discharge after total thyroidectomy: Analysis of Australian data and management recommendations. ANZ J Surg. 2007; 77(4): 199-202. 
17. Terkawi AS, Al-Dhahri SF: Perioperative parathyroid hormone measurements in thyroid surgery: One stone to hit three birds. Braz $\boldsymbol{J}$ Otorhinolaryngol. 2014; 80: 551-355.

18. Le TN, Kerr PD, Sutherland DE, Lambert P: Validation of 1-hour post-thyroidectomy parathyroid hormone level in predicting hypocalcemia. J Otolaryngol Head Neck Surg. 2014; 43: 5.

19. Lecerf P, Orry D, Perrodeau E, Lhommet C, Charretier C, Mor C, Valat C, Bourlier P, de Calan L: Parathyroid hormone decline 4 hours after total thyroidectomy accurately predicts hypocalcemia. Surgery. 2012; 152(5): 863-868.

20. Edafe O, Balasubramanian SP: Incidence, prevalence and risk factors for post-surgical hypocalcaemia and hypoparathyroidism. Gland Surg. 2017; 6(1): S59-S68.

21. Puzziello A, Gervasi R, Orlando G, Innaro $\mathrm{N}$, Vitale M, Sacco R: Hypocalcaemia after total thyroidectomy: Could intact parathyroid hormone be a predictive factor for transient postoperative hypocalcemia? Surgery. 2015; 157(2): 344-8. 